\title{
ANÁLISE EXERGÉTICA DE UM SISTEMA DE REFRIGERAÇÃO POR ABSORÇÃO DE SIMPLES EFEITO OPERANDO COM O PAR LiBr/H $\mathrm{H}_{2} \mathrm{O}$
}

\author{
Igor Marcel Gomes Almeida \\ Engenheiro Mecânico, Especialização em Termociências, Mestrando em Engenharia \\ Mecânica. Departamento de Refrigeração e Climatização. Instituto Federal de Educação, \\ Ciência e Tecnologia do Rio Grande do Norte, Campus Santa Cruz. E-mail: \\ igor.almeida@cefetrn.br

\section{Cleiton Rubens Formiga Barbosa} \\ Engenheiro Mecânico, Doutor em Engenharia Mecânica. Núcleo de Máquinas e Sistemas \\ Térmicos (NMST), Departamento de Engenharia Mecânica, Universidade Federal do Rio \\ Grande do Norte. E-mail: cleiton@ufrnet.br \\ Francisco de Assis Oliveira Fontes \\ Engenheiro Mecânico, Doutor em Engenharia Química, Núcleo de Máquinas e Sistemas \\ Térmicos (NMST), Departamento de Engenharia Mecânica, Universidade Federal do Rio \\ Grande do Norte. E-mail: franciscofontes@uol.com.br
}

\begin{abstract}
RESUMO
A segunda lei da termodinâmica trata da qualidade da energia. Mais especificamente, trata da degradação de energia durante um processo, a geração de entropia, e as perdas de oportunidade da realização de trabalho, o que oferece muita margem para melhorias. $\mathrm{O}$ objetivo deste trabalho é identificar os principais fatores que afetam o desempenho de um sistema de refrigeração por absorção de simples efeito (chiller) que utiliza o par água/brometo de lítio como fluidos refrigerantes e tem como fonte térmica rejeitos provenientes de um grupo motor/gerador elétrico, constituinte de uma planta de cogeração. O sistema tem capacidade de refrigeração entre 4 a 16 TR. A análise exergética foi realizada de modo que a eficácia dos componentes do sistema pôde ser estimada e classificada, permitindo direcionar os esforços no sentido de melhorar e identificar os principais pontos de perda de eficiência no ciclo. As análises revelaram que o gerador e o absorvedor apresentam as maiores fontes de perdas no sistema e os esforços devem ser direcionados de modo a aprimorar o desempenho destes componentes.
\end{abstract}

PALAVRAS-CHAVE: cogeração, refrigeração por absorção, análise exergética, irreversibilidades.

\section{EXERGY ANALYSIS OF AN SINGLE-EFFECT ABSORPTION REFRIGERATION SYSTEM OPERATING WITH THE $\mathrm{LiBr}_{2} \mathrm{H}_{2} \mathrm{O}$ PAIR}

\begin{abstract}
The second law of thermodynamics deals with the quality of energy. More specifically, it is concerned with the degradation of energy during a process, the entropy generation, and the lost opportunities to do work; and it offers plenty of room for improvements. The aim of this paper is identify the main factors affecting the performance of an single-effect absorption refrigeration system (chiller) using lithium bromide/water pair as refrigerants and use waste heat for source from a engine/electric generator, integranf of an cogeneration plant. The refrigeration capacity of system is 4 at $16 \mathrm{TR}$. The exergy analysis was
\end{abstract}


performed so that the effectiveness of the system components could be estimated and ranked, allowing direct efforts to improve and identify the main points of efficiency loss in the cycle. The analysis revealed that the generator and absorber have the largest sources of losses in the system and efforts should be directed to improve the performance of these components.

KEYWORDS: cogeneration, absorption refrigeration, exergy analysis, irreversibilities. 


\section{ANÁLISE EXERGÉTICA DE UM SISTEMA DE REFRIGERAÇÃO POR ABSORÇÃO DE SIMPLES EFEITO OPERANDO COM O PAR LiBr/H $\mathrm{H}_{2} \mathrm{O}$}

\section{INTRODUÇÃO}

A cogeração corresponde à produção simultânea de diferentes formas de energia útil, como as energias eletromecânica e térmica, para suprir as necessidades de uma unidade de processo a partir de uma mesma fonte energética primária, resultando em um aumento da eficiência global do ciclo térmico. Tecnicamente, a cogeração se destaca dentre as demais formas de geração, principalmente por ser uma tecnologia ecológica, apresentando menores índices de emissão de poluentes, ser uma prática cuja tecnologia pode ser facilmente dominada além de garantir maior confiabilidade na geração de insumos energéticos (Balestieri, 2002). Existem diversas configurações de centrais de cogeração, uma das mais utilizadas consiste na interligação de uma unidade geradora de energia elétrica, geralmente um motor de combustão interna e um gerador elétrico, e um sistema de refrigeração por absorção, que aproveita os rejeitos térmicos do motor de combustão para a produção de frio.

A crise energética dos anos 70 e a ênfase continuada em eficiência (conservação dos recursos combustíveis) levou a uma necessidade de revisão completa da forma como os sistemas energéticos podem ser analisados e melhorados termodinamicamente. A nova metodologia é a análise exergética que diz respeito a uma otimização dos componentes do ponto de vista termodinâmico, ou minimização da geração de entropia. Este novo método é baseado na aplicação simultânea da primeira lei e da segunda lei na análise e projeto (Bejan, 2002). O projeto de um sistema energético com baixo custo e alta eficiência é um dos parâmetros essenciais para o desenvolvimento sustentável. Um princípio que é apresentado como eficiente consiste na exergia, trata-se de uma ferramenta que revela as oportunidades de se obter alta eficiência aliada ao baixo custo de desenvolvimento (ElSayed, 2002).

Na engenharia termodinâmica atual, grande ênfase tem sido dada na identificação de mecanismos e componentes de sistemas que são responsáveis por perdas termodinâmicas, dimensão destas perdas (análise exergética), meios de minimização das perdas do sistema (minimização da geração de entropia) e minimização dos custos totais associados com a construção e operação do sistema energético (Bejan, 2002).

De acordo com Stegou-Sagia e Paignigiannis (2003) perdas de exergia são inevitáveis devido a todos os processos naturais serem irreversíveis. Tecnicamente e economicamente falando, a exergia é valiosa e, como consequiência, sempre que se tenta resolver um problema através da aplicação da análise exergética, espera-se mensurar uma perda específica contribuindo para a redução dos custos operacionais. Ainda dentro deste contexto, as decisões acerca da utilização de recursos e dos projetos técnicos eram tradicionalmente baseadas nos parâmetros convencionais de performance, economia, confiabilidade e segurança. Nas décadas recentes, novos parâmetros como danos ambientais e escassez de recursos têm ingressado na lista para formulação de decisões concretas. Porém, os conceitos de eficiências e perdas têm sido também importantes (Rosen, 2002).

Do ponto de vista específico dos sistemas de refrigeração, os processos termodinâmicos envolvidos se configuram com a ocorrência da troca de grandes quantidades de calor com 
o meio. A transferência de calor entre o sistema e o ambiente que ocorre a uma diferença de temperatura finita é a maior causa de irreversibilidades no ciclo. A primeira lei da termodinâmica inclui unicamente a conservação de energia e não fornece informações de como a performance do sistema é degradada, esta lei fornece somente uma ferramenta necessária para a quantificação da energia durante um processo e não oferece a possibilidade de avanços de engenharia (Yumrutaş et al., 2002). A segunda lei, no entanto, trabalha com a qualidade da energia, esta lei se apresenta como uma ferramenta muito eficaz na otimização de sistemas termodinâmicos complexos. Mais especificamente, está centrada na degradação da energia durante um processo, a geração de entropia e a perda de oportunidades de realização de trabalho (Çengel e Boles, 2001).

Já é consolidado na literatura que os sistemas de refrigeração por absorção apresentam coeficiente de performance (COP) muito baixo, entre 0,4 a 0,8 para sistemas de simples efeito. Desta forma, diversas pesquisas têm sido realizadas no intuito de localizar as principais fontes de perda de eficiência destes sistemas. A análise pela segunda lei da termodinâmica (exergia) tem sido a principal aliada para a busca do aperfeiçoamento destes ciclos, a qual está preocupada com a degradação da energia, a geração de entropia e, consequentemente, as perdas de oportunidade da realização de trabalho, identificando os pontos críticos que requerem os esforços principais no sentido de aprimorar a performance de sistemas. O método da exergia, conhecido como análise pela segunda lei, calcula a perda de exergia causada pelas irreversibilidades, sendo assim uma importante propriedade termodinâmica, pois indica o trabalho útil que pode ser produzido por uma substância ou a quantidade de trabalho necessária para concluir um determinado processo, fornecendo informações a respeito da ocorrência de ineficiências do sistema (Talbi e Agnew, 2002).

Aphornratana e Eames (1995) foram os pioneiros na utilização da análise exergética e desenvolveram uma metodologia de fácil aplicação para o uso da análise pela segunda lei em ciclos de refrigeração por absorção de simples efeito que utilizam o par água/brometo de lítio $\left(\mathrm{H}_{2} \mathrm{O} / \mathrm{LiBr}\right)$. Os autores apresentam ainda um método simples para determinação da entropia de soluções de água/brometo de lítio. Talbi e Agnew (2002) calcularam as perdas de exergia inerentes a um sistema de refrigeração por absorção de simples efeito utilizando água/brometo de lítio, os autores concluíram que as trocas de calor à temperaturas consideravelmente superiores a ambiente são as maiores fontes de de perda de exergia nos componentes do sistema. $\mathrm{O}$ absorvedor apresentou a maior taxa de perda de exergia, cerca de 59\%. Lee e Sherif (2001) analisaram com base na primeira e segunda lei da termodinâmica um sistema de absorção utilizando água/brometo de lítio para aplicações de aquecimento e resfriamento. Os autores determinaram o COP e a eficiência exergética do sistema sob diferentes condições de operação e avaliaram os fatores de maior influência na performance do ciclo. Fornecem ainda uma metodologia para determinação da entropia e exergia de soluções de $\mathrm{H}_{2} \mathrm{O} / \mathrm{LiBr}$. Sencan et al. (2005) aplicaram o método da exergia em um ciclo de absorção de simples efeito operando com $\mathrm{H}_{2} \mathrm{O} / \mathrm{LiBr}$, o autores avaliaram a perda de exergia, entalpia, entropia, temperatura, vazão mássica e fluxo de calor em cada componente do sistema. Os resultados obtidos mostraram que as perdas de exergia no condensador e evaporador são menores que no gerador e no absorvedor. Os autores atribuem este fato ao calor envolvido durante a mistura das soluções que ocorrem nestes componentes e que não está presente nos fluidos puros, como é o caso do estado dos fluidos no condensador e evaporador. Kilic e Kaynakli (2007) citam que a aplicação da segunda lei da termodinâmica em sistemas de refrigeração por absorção facilita a identificação de componentes com grande perda de exergia, permitindo direcionar os esforços de se obter sistemas com alta eficiência da segunda lei. 
Neste sentido, com base nos trabalhos teóricos e computacionais disponíveis na literatura, o objetivo deste trabalho é aplicar o método da exergia em um chiller de absorção de simples efeito para fins de climatização a partir de rejeitos térmicos de uma unidade de cogeração. A análise será realizada em cada componente do sistema, estimando as perdas de exergia que existem no ciclo com o auxílio de uma plataforma desenvolvida no EES (Engineering Equation Solver) para determinação das propriedades termodinâmicas da solução $\mathrm{H}_{2} \mathrm{O} / \mathrm{LiBr}$ em todo o ciclo. Este artigo consiste em um estudo de caso da análise exergética aplicada a sistemas de refrigeração por absorção.

\section{DESCRIÇÃO DO SISTEMA DE REFRIGERAÇÃO POR ABSORÇÃO}

A central de cogeração é composta basicamente por um grupo gerador movido a gás natural, caldeira de recuperação de calor dos gases de combustão, trocador de calor para a água das camisas do motor, sistema de refrigeração por absorção de simples estágio de 16 TR (capacidade máxima), caldeira a gás natural, torre de arrefecimento de água e outros componentes auxiliares como bombas, trocadores de calor, válvulas e torre de arrefecimento. A central opera no regime topping, no qual a energia elétrica é gerada com prioridade sobre a térmica. O calor dos gases de exaustão e das camisas do motor é recuperado a partir de uma caldeira recuperadora de calor que fornece calor necessário para acionamento do chiller, obtendo-se água gelada que em seguida é direcionada para um sistema de condicionamento de ar do tipo fan-coil. O sistema de refrigeração por absorção utiliza água quente como fonte térmica para o gerador. A figura 1 apresenta a configuração utilizada na central de cogeração.

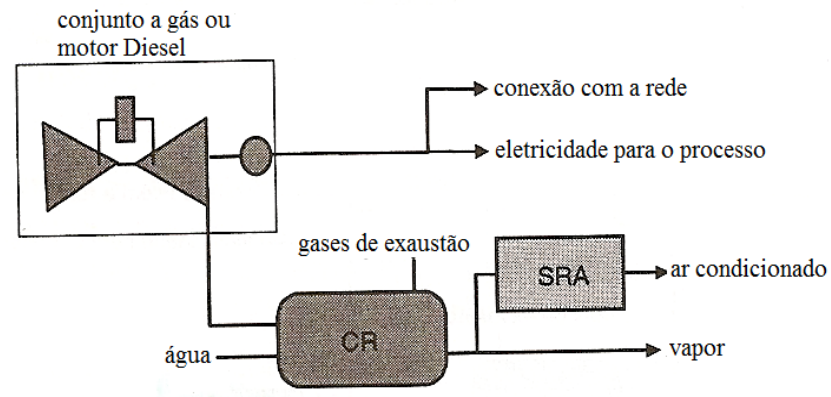

Figura 1 - Configuração da central de cogeração, com sistema de refrigeração por absorção (SRA) e caldeira de recuperação de calor (CR). Fonte: Balestieri, 2002.

O objetivo da presente análise é aplicar o método da exergia apenas ao sistema de refrigeração da central de cogeração. Uma representação do ciclo de refrigeração proposto é mostrada na figura 2 a seguir. 


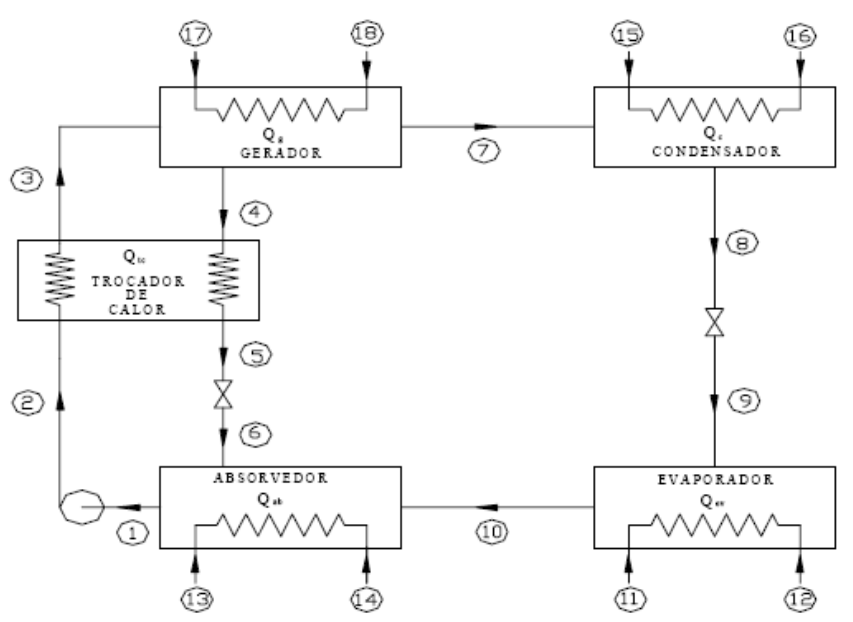

Figura 2 - Representação do sistema de refrigeração por absorção.

O sistema é composto por bomba de circulação da solução, trocador de calor, gerador, absorvedor, condensador, evaporador e válvulas de expansão. Observando a figura 2, os pontos 17 e 18 se referem ao fornecimento e rejeição da água quente no gerador, proveniente da caldeira de recuperação de calor. No absorvedor, condensador e evaporador utiliza-se água como meio de troca de calor. A água utilizada previamente no absorvedor é em seguida fornecida ao condensador, sendo depois enviada à torre de arrefecimento. No evaporador utiliza-se água fria, obtendo-se a diminuição de temperatura desta e seu posterior fornecimento ao circuito de ar condicionado (sistema fan-coil). A tabela 1 apresenta os pontos característicos do ciclo e seus parâmetros funcionais proposto por Santos et al. (2005). Onde são fornecidos os dados de pressão dos fluidos $\mathbf{P}(\mathrm{kPa})$, temperatura $\mathbf{T}\left({ }^{\circ} \mathrm{C}\right)$, vazão mássica $\mathbf{m}(\mathrm{kg} / \mathrm{s})$ e concentração da mistura $\mathbf{x}(\%)$.

Tabela 1: Pontos característicos do sistema de refrigeração por absorção.

\begin{tabular}{|c|c|c|c|c|c|c|c|c|c|}
\hline Ponto & $\mathbf{P}(\mathbf{k P a})$ & $\mathbf{T}\left({ }^{\mathbf{C}} \mathbf{C}\right)$ & $\mathbf{m}(\mathbf{k g} / \mathbf{s})$ & $\mathbf{x}(\mathbf{\%})$ & $\mathbf{P o n t o}$ & $\mathbf{P}(\mathbf{k P a})$ & $\mathbf{T}\left({ }^{\mathbf{0}} \mathbf{C}\right)$ & $\mathbf{m} \mathbf{( k g} / \mathbf{s})$ & $\mathbf{x}(\mathbf{\%})$ \\
\hline $\mathbf{1}$ & 0,87 & 34,44 & 0,05473 & 54,9 & $\mathbf{1 0}$ & 0,87 & 5 & 0,007782 & - \\
\hline $\mathbf{2}$ & 6,275 & 34,44 & 0,05473 & 54,9 & $\mathbf{1 1}$ & 101,3 & 12 & 0,8772 & - \\
\hline $\mathbf{3}$ & 6,275 & 63,6 & 0,05473 & 54,9 & $\mathbf{1 2}$ & 101,3 & 7 & 0,8772 & - \\
\hline $\mathbf{4}$ & 6,275 & 91,13 & 0,04695 & 64,0 & $\mathbf{1 3}$ & 101,3 & 29,5 & 1,823 & - \\
\hline $\mathbf{5}$ & 6,275 & 52,13 & 0,04695 & 64,0 & $\mathbf{1 4}$ & 101,3 & 32,45 & 1,823 & - \\
\hline $\mathbf{6}$ & 0,87 & 52,13 & 0,04695 & 64,0 & $\mathbf{1 5}$ & 101,3 & 32,45 & 1,823 & - \\
\hline $\mathbf{7}$ & 6,275 & 80,59 & 0,007782 & - & $\mathbf{1 6}$ & 101,3 & 35 & 1,823 & - \\
\hline $\mathbf{8}$ & 6,275 & 37 & 0,007782 & - & $\mathbf{1 7}$ & 101,3 & 300 & 0,2143 & - \\
\hline $\mathbf{9}$ & 0,87 & 5 & 0,007782 & 32,0 & $\mathbf{1 8}$ & 101,3 & 200 & 0,2143 & - \\
\hline
\end{tabular}




\section{ANÁLISE TERMODINÂMICA}

A primeira lei da termodinâmica afirma que a energia é conservada, desta forma, não demonstra a ocorrência de irreversibilidades em um sistema, como a originada, por exemplo, pela perda de pressão do fluido refrigerante. De acordo com a primeira lei, todas as formas de energia são convertidas em outras. No entanto, a segunda lei impõe limitações a essa conversão de energia. Todos os processos reais são irreversíveis, devido a alguns fatores, como:

- Atrito fluido, dissipando trabalho na forma de calor;

- Transferência de calor de um corpo a alta temperatura para um corpo a baixa temperatura;

- Expansão e perda de pressão em processos adiabáticos;

Neste artigo, o modelo matemático adotado é o proposto por Aphornratana e Eames (1995). As propriedades termodinâmicas dos fluidos de trabalho em todos os pontos do ciclo (ver tabela 1) foram obtidas mediante modelo computacional proposto por Talbi e Agnew (2002) e Chua et al. (2000), utilizando o software EES (Engineering Equation Solver). A tabela 2 apresenta as propriedades termodinâmicas obtidas a partir da simulação para todos os pontos do ciclo. As propriedades termodinâmicas obtidas consistiram na entalpia h $(\mathrm{kJ} / \mathrm{kg})$ e entropia $\mathbf{s}(\mathrm{kJ} / \mathrm{kgK})$.

Tabela 2: Propriedades termodinâmicas do sistema de refrigeração por absorção.

\begin{tabular}{|c|c|c|c|c|c|c|c|c|c|}
\hline Ponto & $\begin{array}{c}\mathbf{P} \\
(\mathbf{k P a})\end{array}$ & $\begin{array}{c}\mathbf{T} \\
\left({ }^{\mathbf{}} \mathbf{C}\right)\end{array}$ & $\begin{array}{c}\mathbf{h} \\
(\mathbf{k J} / \mathbf{k g})\end{array}$ & $\begin{array}{c}\mathbf{S} \\
(\mathbf{k J} / \mathbf{k g K})\end{array}$ & $\mathbf{P o n t o}$ & $\begin{array}{c}\mathbf{P} \\
(\mathbf{k P a})\end{array}$ & $\begin{array}{c}\mathbf{T} \\
\left({ }^{\mathbf{}} \mathbf{C}\right)\end{array}$ & $\begin{array}{c}\mathbf{h} \\
(\mathbf{k J} / \mathbf{k g})\end{array}$ & $\begin{array}{c}\mathbf{S} \\
(\mathbf{k J} / \mathbf{k g K})\end{array}$ \\
\hline $\mathbf{1}$ & 0,87 & 34,44 & 81,847 & 0,20895 & $\mathbf{1 0}$ & 0,87 & 5 & 2511,0 & 9,0270 \\
\hline $\mathbf{2}$ & 6,275 & 38,21 & 89,651 & 0,22312 & $\mathbf{1 1}$ & 101,3 & 12 & 50,49 & 0,1805 \\
\hline $\mathbf{3}$ & 6,275 & 63,6 & 142,277 & 0,41230 & $\mathbf{1 2}$ & 101,3 & 7 & 29,48 & 0,1062 \\
\hline $\mathbf{4}$ & 6,275 & 91,13 & 232,396 & 0,49650 & $\mathbf{1 3}$ & 101,3 & 29,5 & 123,8 & 0,4299 \\
\hline $\mathbf{5}$ & 6,275 & 52,13 & 161,484 & 0,26341 & $\mathbf{1 4}$ & 101,3 & 32,45 & 136,1 & 0,4705 \\
\hline $\mathbf{6}$ & 0,87 & 52,13 & 161,484 & 0,26341 & $\mathbf{1 5}$ & 101,3 & 32,45 & 136,1 & 0,4705 \\
\hline $\mathbf{7}$ & 6,275 & 80,59 & 2651,0 & 8,56300 & $\mathbf{1 6}$ & 101,3 & 35 & 146,7 & 0,5052 \\
\hline $\mathbf{8}$ & 6,275 & 37 & 155,1 & 0,53220 & $\mathbf{1 7}$ & 101,3 & 300 & 3074,0 & 8,2100 \\
\hline $\mathbf{9}$ & 0,87 & 5 & 155,1 & 0,53220 & $\mathbf{1 8}$ & 101,3 & 200 & 2875,0 & 7,8280 \\
\hline
\end{tabular}




\section{EXERGIA}

É definida como a máxima quantidade de trabalho reversível que pode ser obtida por um fluido, e é dada pela equação 1 .

$$
\psi=\left(h-h_{0}\right)-T_{0}\left(s-s_{0}\right)
$$

Os termos $h_{0}$ e $s_{0}$ representam a entalpia e entropia do fluido a uma condição de referência, adotou-se a temperatura ambiente de $25^{\circ} \mathrm{C}$ e pressão de $101,3 \mathrm{kPa}$. A variação de exergia (perda de exergia) é expressa por

$$
\Delta \psi=\sum \dot{m}_{i} \psi_{i}-\sum \dot{m}_{e} \psi_{e}-\dot{Q}\left(1-\frac{T_{0}}{T}\right)-W
$$

Os dois primeiros termos são os somatórios (balanços) de exergia nas entradas e saídas dos componentes do sistema de refrigeração. O terceiro termo é a exergia do calor $Q$ que é transferido a uma temperatura constante $T$. A exergia do calor é igual ao trabalho obtido por um motor de Carnot operando entre $T$ e $T_{0}$. O último termo é o trabalho mecânico transferido para o sistema ou pelo sistema.

\section{BALANÇO DE ENERGIA E EXERGIA NOS COMPONENTES}

Para cada componente do sistema de absorção mostrado na figura 2, os balanços de energia e exergia são apresentados abaixo.

\section{Condensador}

$$
\begin{aligned}
& \dot{Q}_{\text {con }}=\dot{m}_{7}\left(h_{7}-h_{8}\right)=\dot{m}_{15}\left(h_{16}-h_{15}\right) \\
& \Delta \psi_{\text {con,água }}=\dot{m}_{15}\left(\psi_{16}-\psi_{15}\right)
\end{aligned}
$$

Considera-se que o calor $Q_{c o n}$ é rejeitado para a água de resfriamento a uma temperatura constante $T_{c o n}$. A irreversibilidade que ocorre durante a troca de calor é mensurada pela diferença entre a exergia do fluxo de calor $Q_{c o n}$ e a variação de exergia na água de resfriamento:

$$
\Delta \psi_{\text {con,calor }}=\dot{Q}_{\text {con }}\left(1-\frac{T_{0}}{T_{c o n}}\right)-\dot{m}_{15}\left(\psi_{16}-\psi_{15}\right)
$$

A irreversibilidade interna é definida como a diferença entre a variação de exergia do fluido de trabalho (refrigerante ou solução) e a exergia do fluxo de calor $Q_{c o n}$ :

$$
\Delta \psi_{\text {con, in }}=\dot{m}_{7}\left(\psi_{7}-\psi_{8}\right)-\dot{Q}_{\text {con }}\left(1-\frac{T_{0}}{T_{\text {con }}}\right)
$$

Para os demais componentes, os balanços de energia e exergia são obtidos de forma similar, as equações são demonstradas abaixo. 


\section{Evaporador}

$\dot{Q}_{\text {evap }}=m_{9}\left(h_{9}-h_{10}\right)=m_{11}\left(h_{12}-h_{11}\right)$

equação(7)

$\Delta \psi_{\text {evap }, \text { calor }}=\dot{Q}_{\text {evap }}\left(1-\frac{T_{0}}{T_{\text {evap }}}\right)-\dot{m}_{11}\left(\psi_{12}-\psi_{11}\right)$

$\Delta \psi_{\text {evap, in }}=\dot{m}_{9}\left(\psi_{9}-\psi_{10}\right)-\dot{Q}_{\text {evap }}\left(1-\frac{T_{0}}{T_{\text {evap }}}\right)$

equação(9)

$\Delta \psi_{\text {evap,água }}=m_{11}\left(\psi_{12}-\psi_{11}\right)$

equação(10)

\section{Absorvedor}

$Q_{a b s}=m_{10} h_{10}+m_{6} h_{6}-m_{1} h_{1}=m_{13}\left(h_{14}-h_{13}\right)$

equação(11)

$\Delta \psi_{a b s, c a l o r}=\dot{Q}_{a b s}\left(1-\frac{T_{0}}{T_{a b s}}\right)-m_{13}\left(\psi_{14}-\psi_{13}\right)$

equação(12)

$\Delta \psi_{a b s, i n}=\dot{m}_{10} \psi_{10}+\dot{m}_{6} \psi_{6}-\dot{m}_{1} \psi_{1}-Q_{a b s}\left(1-\frac{T_{0}}{T_{a b s}}\right)$

equação(13)

$\Delta \psi_{\text {ab,água }}=m_{13}\left(\psi_{14}-\psi_{13}\right)$

equação(14)

\section{Gerador}

$\dot{Q}_{\text {ger }}=m_{3} h_{3}-m_{4} h_{4}-m_{7} h_{7}=m_{17}\left(h_{17}-h_{18}\right)$

equação(15)

$\Delta \psi_{\text {ger,calor }}=\dot{Q}_{\text {ger }}\left(1-\frac{T_{0}}{T_{\text {ger }}}\right)-\dot{m}_{17}\left(\psi_{17}-\psi_{18}\right)$

equação(16)

$\Delta \psi_{\text {ger }, \text { in }}=\dot{m}_{3} \psi_{3}-\dot{m}_{4} \psi_{4}-\dot{m}_{7} \psi_{7}-\dot{Q_{\text {ger }}}\left(1-\frac{T_{0}}{T_{g e r}}\right)$

equação(17)

$\Delta \psi_{\text {ger,água }}=m_{17}\left(\psi_{17}-\psi_{18}\right)$

equação(18) 
Trocador de calor (solução)

$\Delta \psi_{t c, \text { calor }}=m_{4} \psi_{4}+m_{2} \psi_{2}-m_{3} \psi_{3}-m_{5} \psi_{5}$

equação(19)

Válvula de expansão (refrigerante)

$\Delta \psi_{\text {valv, ref }}=m_{8}\left(\psi_{8}-\psi_{9}\right)$

equação(20)

Razão de circulação da solução

$S C R=\frac{m_{1}}{m_{10}}$

equação(21)

\section{RESULTADOS E DISCUSSÕES}

Aplicando as equações e propriedades termodinâmicas do sistema em uma plataforma computacional do software EES, obtiveram-se os resultados mostrados nas tabelas abaixo. Para simplificar a simulação e análise da transferência de calor entre o sistema e o ambiente o trabalho fornecido à bomba de circulação da solução e as perdas por atrito no sistema foram negligenciados. A tabela 3 apresenta os resultados obtidos pela aplicação da primeira lei da termodinâmica nos principais componentes do sistema.

Tabela 3: Fluxos de energia nos componentes do sistema.

\begin{tabular}{|c|c|c|}
\hline Componente & Símbolo & Fluxo de energia (kW) \\
\hline Gerador & $\mathrm{Q}_{\mathrm{g}}$ & 23,755 \\
\hline Condensador & $\mathrm{Q}_{\mathrm{c}}$ & 19,432 \\
\hline Evaporador & $\mathrm{Q}_{\mathrm{e}}$ & 18,333 \\
\hline Absorvedor & $\mathrm{Q}_{\mathrm{a}}$ & 22,422 \\
\hline Coeficiente de Performance & $\mathrm{COP}$ & 0,771 \\
\hline
\end{tabular}

A análise exergética foi realizada em cada componente. As perdas de exergia são obtidas utilizando basicamente as Eqs. (1) e (2). As perdas de exergia nos vários componentes do ciclo são dadas na tabela 4. A perda de exergia é uma quantidade de disponibilidade consumida em um determinado processo. As irreversibilidades em sistemas de absorção reduzem o $\mathrm{COP}$ e a eficiência exergética a valores abaixo de um ponto ideal devido às diversas "não-idealidades"que existem nos processos. Esta análise enfatiza como as perdas e irreversibilidades impactam a performance do sistema, sendo uma importante ferramenta de avaliação e aperfeiçoamento do projeto dos componentes dos sistemas de refrigeração por absorção. A exergia fornecida representada na coluna 2 da tabela 4 diz respeito ao somatório das exergias dos fluxos que entram em cada componente (volume de controle). A exergia recuperada consiste no somatório das exergias dos fluxos que saem de cada componente. 
Tabela 4: Perdas de exergia do sistema de refrigeração por absorção.

\begin{tabular}{|c|c|c|c|}
\hline Componente & $\begin{array}{c}\text { Exergia fornecida } \\
(\mathbf{k W})\end{array}$ & $\begin{array}{c}\text { Exergia recuperada } \\
(\mathbf{k W})\end{array}$ & $\begin{array}{c}\text { Perda de exergia } \\
(\mathbf{k W})\end{array}$ \\
\hline Gerador & 135,376 & 118,403 & 16,973 \\
\hline Condensador & 1,438 & 1,111 & 0,327 \\
\hline Evaporador & 1,021 & 0,6474 & 0,373 \\
\hline Absorvedor & $-0,7257$ & $-2,409$ & 1,683 \\
\hline $\begin{array}{c}\text { Válvula de } \\
\text { expansão }\end{array}$ & 0,00746 & 0,00746 & - \\
\hline Trocador de calor & 0,6083 & 0,3350 & 0,273 \\
\hline Sistema & 137,725 & 118,094 & 19,631 \\
\hline
\end{tabular}

Nos sistemas de absorção, as principais fontes de irreversibilidades são as relacionadas aos processos de mistura de dois fluidos que ocorrem no absorvedor e no gerador, com a evaporação do refrigerante no gerador a uma determinada concentração, que requer uma grande quantidade de calor para evaporar a um estado puro. O superaquecimento excessivo da água que ocorre no gerador se constitui como uma fonte de perda termodinâmica, levando à necessidade de uma maior quantidade de resfriamento no condensador. De acordo com a tabela 4, o absorvedor e o gerador apresentaram as maiores perdas de exergia justamente devido às perdas decorrentes do processo de mistura e separação dos fluidos nestes componentes. Segundo Sencan et al. (2005) estas perdas podem ser reduzidas mediante a aplicação de uma fonte de calor com uma temperatura mínima necessária aos processos de absorção e dessorção nestes componentes. Desta forma, é necessária a avaliação das características termodinâmicas e químicas das misturas para se obter uma temperatura mínima da fonte de calor que realize estes processos com a maior eficiência possível.

As perdas que ocorrem no absorvedor são também ocasionadas pela diferença de temperatura entre este componente e o ambiente. De acordo com Talbi e Agnew (2002), uma forma de reduzir esta fonte de perda é proporcionar um aumento na área de troca de calor do absorvedor. Aphornratana e Eames (1995) citam que estas irreversibilidades podem ser reduzidas através da diminuição da razão de circulação da solução e uso de um trocador de calor (solução) de maior efetividade, limitada pela temperatura de cristalização da solução. A razão de circulação da solução é reduzida mediante o aumento da concentração da solução no absorvedor. Esta concentração aumenta com o aumento da temperatura ou da pressão, e vice-e-versa. $\mathrm{O}$ aumento da temperatura no gerador resulta em uma razão de circulação da solução menor. No entanto, isto não reduzirá as irreversibilidades no absorvedor, pois as irreversibilidades internas aumentam com o aumento da temperatura da solução que entra no absorvedor. Kandlikar apud Aphornratana e Eames (1995) afirma que o uso do calor rejeitado durante o processo de absorção para pré-aquecer a solução antes da sua entrada no trocador de calor é um método eficiente de redução da perda de exergia no absorvedor. Kaushik e Arora (2009) afirmam que a eficiência exergética do sistema aumenta consideravelmente com o aumento da temperatura do gerador, tendendo a declinar com a progressão do aumento desta temperatura. As perdas que ocorrem no condensador resultam da existência do vapor de 
água superaquecido que provém do gerador e entra no condensador, onde em seguida ocorre a transferência de calor a uma temperatura relativamente baixa.

A considerável perda de exergia que ocorre no evaporador é ocasionada principalmente pela diferença de temperatura entre o ambiente e a temperatura de evaporação do refrigerante. As irreversibilidades associadas à transferência de calor no evaporador, tem efeito muito importante na performance do sistema. De acordo com Aphornratana e Eames (1995), para se obter uma melhor performance do ciclo de refrigeração por absorção, a prioridade deve ser dada ao evaporador, seguido do absorvedor. Não foram consideradas as perdas de exergia decorrentes da válvula de expansão (solução) e bomba de circulação da solução, pois as perdas nestes componentes são muito pequenas comparadas às perdas do sistema (Kilic e Kaynakli, 2007).

\section{CONCLUSÕES}

É fato consumado que sistemas de refrigeração por absorção apresentam penalização da performance em relação aos sistemas de compressão de vapor. No entanto, este tipo de ciclo pode ser atrativo na aplicação integrada a sistemas com coletores solares, cogeração e trigeração. Desta forma, diversas pesquisas têm sido realizadas no intuito de localizar as principais fontes de perda de eficiência destes sistemas. A análise pela segunda lei da termodinâmica (exergia) tem sido a principal aliada para a busca do aperfeiçoamento destes ciclos.

A análise desenvolvida neste estudo pôde mostrar que os principais esforços no sentido de aperfeiçoamento dos sistemas de refrigeração por absorção devem se concentrar no desenvolvimento de componentes mais eficientes. Destacando-se principalmente o gerador, absorvedor e evaporador, que apresentaram as maiores perdas de exergia em todo o sistema.

A ocorrência das perdas de exergia nos trocadores de calor do sistema (condensador e evaporador) são principalmente influenciadas pela diferença de temperatura entre estes componentes e a ambiente.

Do ponto de vista das irreversibilidades no gerador e absorvedor, estas podem ser reduzidas a valores aceitáveis mediante projeto de um trocador de calor da solução com alta efetividade, além de dimensionar o ciclo para operar com baixa razão de circulação da solução (SCR), devendo-se tentar reduzir este parâmetro para valores inferiores a 7,0, de acordo com Aphornratana e Eames (1995). Outro fator que influencia consideravelmente na elevada perda de exergia no gerador são as elevadas temperaturas de fornecimento de calor para o sistema, deve-se estimar valores ótimos de temperatura da fonte térmica para maior eficiência do gerador.

\section{REFERÊNCIAS BIBLIOGRÁFICAS}

1. BALESTIERI, J.A.P., Cogeração: geração combinada de eletricidade e calor. Capítulo 1. Florianópolis: Ed. da UFSC, 2002.

2. TALBI, M.M., AGNEW, B.; Exergy analysis: an absorption refrigerator using lithium bromide and water as the working fluids. Applied Thermal Engineering, vol.20, pp. 619-630, 2000. 
3. APHORNRATAN, S., EAMES, I.W., Thermodynamics analysis of absorption refrigeration cycles using the second law of thermodynamics method. International Journal of Refrigeration, vol.18, pp. 244-252, 1995.

4. LEE, S.F., SHERIF, S.A., Thermodynamic analysis of a lithium bromide/water absorption system for cooling and heating applications. International Journal of Energy Research, vol. 25, pp. 1019-1031, 2001.

5. SENCAN, A., YAKUT, K.A., KALOGIROU, S.A., Exergy analysis of lithium bromide/water absorption systems. Renewable Energy, vol.30, pp. 645-657, 2005.

6. KILIC, M., KAYNAKLI, O., Second law-based thermodynamic analysis of waterlithium bromide absorption refrigeration system. Energy, vol.32, pp.1505-1512, 2007.

7. SANTOS, P.H.D., VARANI, C.M.R., SANTOS, C.A.C., MOREIRA, H.L., Dimensionamento de um sistema de refrigeração por absorção de simples efeito, água-brometo de lítio, com gás natural como fonte térmica. IX Congresso Brasileiro de Refrigeração, Ar condicionado, Aquecimento e Tratamento de ar. São Paulo, 2005.

8. CHUA, H.T., TOH, H.K., MALEK, A., NG, K.C., SRINIVASAN, K., Improved thermodynamic property field of $\mathrm{LiBr}-\mathrm{H} 2 \mathrm{O}$ solution. International Journal of Refrigeration 23:412-429. 2000.

9. KAUSHIK, S.C., ARORA, A., Energy and exergy analysis of single effect and series flow double effect water-lithium bromide absorption refrigeration systems. International Journal of Refrigeration, doi:10.1016/j.ijrefrig.2009.01.017. 2009.

10. BEJAN, A., Fundamentals of exergy analysis, entropy generation minimization, and the generation of flow architecture. International Journal of Energy Research, 26: 545-565. 2002.

11. EL-SAYED, Y.M., Application of exergy to design. Energy Conversion and Management, 43: 1165-1185. 2002.

12. STEGOU-SAGIA, A., PAIGNIGIANNIS, N., Exergy losses in refrigeration systems: A study for performance comparisons in compressor and condenser. International Journal of Energy Research 27:1067-1078. 2003.

13. ROSEN, M.A., Clarifying thermodynamic efficiencies and losses via exergy. Exergy, An international Journal 2: 3-5. 2002.

14. YUMRUTAS, R., KUNDUZ, M., KANOGLU, M., Exergy analysis of vapor compression refrigeration systems. Exergy, An international Journal 2:266-272.

15. ÇENGEL, Y., BOLES, M., Thermodynamics: An engineering approach. $4^{\text {th }}$ Edition. McGraw-Hill Science. 1056 pages. 2001. 